Maria de FÁtima Rezende Francisco'

ROSIANE MATTAR ${ }^{2}$

FÁtima Ferreira Bortoletti'

MARY UCHIYAMA NAKAMURA ${ }^{2}$

\title{
Sexualidade e depressão em gestantes com aborto espontâneo de repetição
}

\author{
Sexuality and depression among pregnant \\ women with recurrent spontaneous abortion
}

Artigo Original

\author{
Palavras-chave \\ Aborto espontâneo \\ Sexualidade \\ Depressão \\ Gestantes \\ Questionários \\ Keywords \\ Abortion, spontaneous \\ Sexuality \\ Depression \\ Pregnant women \\ Questionnaires
}

Correspondêncio

Maria de Fótima Rezende Francisco

Rua Napoleão de Barros, 875 - Vila Clementino

CEP: 04024-002

São Paulo (SP), Brasil

Recebido

$17 / 12 / 2013$

Aceito com modificacọoes

$27 / 01 / 2014$

\section{Resumo}

OBJETIVO: Comparar os sintomas de depressão e o comportamento sexual de gestantes com histórico de aborto espontâneo de repetição (AER) com gestantes que não vivenciaram AER. MÉTODOS: Trata-se de um estudo prospectivo caso-controle. O primeiro grupo é formado por mulheres que apresentaram AER e o segundo, por primigestas. Foram utilizados o Beck Depression Inventory (BDI), o Female Sexual Function Index (FSFI) e mais um questionário desenvolvido pelos autores sobre aspectos emocionais decorrentes da relação sexual na gravidez. Para comparar as variáveis quantitativas, com distribuição normal, utilizou-se o teste t de Student, e as variáveis categóricas foram comparadas com base no teste do $\chi^{2}$ ou no teste exato de Fisher. A significância estatística foi considerada para valores de $p<0,05$. RESULTADOS: $\bigcirc$ BDI indicou frequência aproximadamente duas vezes maior de depressão no Grupo AER (19,9 versus 10,0\%, respectivamente). Quanto à função sexual, os escores médios do FSFI foram de 21, 1 e 16,4 (p<0,05), respectivamente nos Grupos AER e

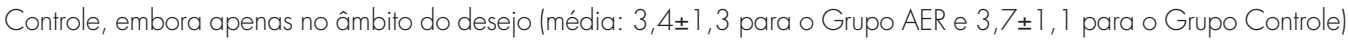
não houvesse diferença significante $(p=0,1)$. Observamos que, independentemente de a gestante ter ou não histórico de AER, quanto maior o escore de depressão, menor o escore de sexualidade (r=-0,3). CONCLUSÕES: Gestantes do Grupo AER apresentam depressão com frequência duas vezes mais elevada e função sexual mais comprometida. Há associação inversa entre depressão e função sexual.

\section{Abstract}

PURPOSE: It was to compare pregnant women who experienced recurrent spontaneous abortion (RSA) and those who did not in terms of the prevalence of depressive symptoms and sexual behavior. METHODS: A prospective case-control study was carried out. The first group consisted of women with RSA and the second, of primigravidae. The Beck Depression Inventory (BDI), the Female Sexual Function Index (FSFI) and one more questionnaire, developed by the authors themselves, about emotional aspects resulting from sexual intercourse during pregnancy were applied. The Student t-test was used to compare quantitative variables with normal distribution, and categorical variables were compared by the chi-square test or Fisher's exact test. The level of significance was set at $p<0.05$. RESULTS: The BDI showed (19.9 versus 10.0\%) approximately twice the incidence of depression in the RSA group. Regarding sexual function, the average scores of the FSFI were 21.1 and $16.4(p<0.05)$ for the study and control groups, respectively, although no significant difference was observed only in the desire domain laverage 3.4 1.3 for the RSA group and 3.7 \pm 1.1 for control group) ( $p=0.1)$. We observed that, regardless the presence or absence of an RSA history among the pregnant women, the higher the depression score, the lower the sexuality score $(r=-0,3)$. CONCLUSIONS: The RSA pregnant group often experiences twice higher depression and more impaired sexual function. There is an inverse association between depression and sexual function.
Departamento de Obstetrícia da Escola Paulista de Medicina da Universidade Federal de São Paulo - UNIFESP - São Paulo (SP), Brasil. Programa de Pós-graduação em Obstetrícia, Escola Paulista de Medicina, Universidade Federal de São Paulo - UNIFESP São Paulo (SP), Brasil.

2Departamento de Obstetrícia, Escola Paulista de Medicina, Universidade Federal de São Paulo - UNIFESP - São Paulo (SP), Brasil. Conflito de interesses: não há. 
Introdução

A gravidez, com ou sem intercorrências, pode trazer sentimentos de perda e de medo, pois é uma nova etapa de vida, e a ambivalência própria desse período muitas vezes traz dificuldades sexuais e de origem psicológica ${ }^{1}$. Os aspectos psíquicos do aborto espontâneo de repetição (AER) podem ser potencializados nas gestações subsequentes, podendo determinar elevada frequência de problemas emocionais, como síndromes depressivas e transtornos da ansiedade ${ }^{2-5}$. Esses sintomas podem, inclusive, perdurar por longos períodos ${ }^{4}$. Estudos comprovam que a perda gestacional espontânea de repetição aumenta o risco de desordem emocional, que é acompanhada em 10 a 33\% dos casos $^{4}$. Entre as mulheres grávidas, 30 a $38 \%$ apresentam sintomas de depressão, embora seja difícil estabelecer um número definitivo, pois muitos dos sintomas apresentados pela gestante se confundem com os da própria gravidez $z^{6}$.

A depressão é bastante comum em todo o mundo. Sua prevalência durante a gravidez foi estimada em $7 \%$ na Austrália e em Taiwan, 8\% no Chile e $9 \%$ no Canadá. Nos Estados Unidos, varia de 7 a $13 \%$ e na Europa, de 7 a $8 \%^{7}$. No Brasil, a prevalência foi de $14,2 \%^{8}$ em estudos desenvolvidos no Rio de Janeiro e de 9,0\% em São Paulo.

O casal com história de abortamento, ao decidir por uma nova gravidez, pode apresentar ansiedade e perda de libido e do desejo sexual em consequência da sensação da obrigatoriedade de engravidar'.

A satisfação sexual, por outro lado, é um item importante na qualidade de vida segundo a Organização Mundial da Saúde (OMS). No entanto, estudos mostram que há elevados índices de disfunção sexual, sendo a prevalência mais alta entre as mulheres (43\%), quando comparadas aos homens (31\%), e que isso se associa a características demográficas e de educação. Essa diferença entre os gêneros pode indicar maior comprometimento de saúde física e emocional das mulheres ${ }^{10}$.

Verificamos carência de trabalhos que associem aspectos emocionais oriundos do AER a comportamento sexual. Por ser o AER uma condição que causa muito sofrimento aos casais, e por ser relevante para a saúde do ser humano, consideramos oportuno desenvolver este estudo.

\section{Métodos}

Este é um estudo prospectivo caso-controle, com mulheres grávidas, com e sem AER, que foram atendidas entre janeiro e dezembro de 2011 no ambulatório de pré-natal de Aborto Espontâneo de Repetição e no de Baixo Risco, na Universidade Federal de São Paulo/Escola Paulista de Medicina (UNIFESP/EPM), localizada na cidade São Paulo. O projeto foi aprovado pelo Comitê de Ética em Pesquisa (CEP) 1270/10 e todas as participantes assinaram um termo de consentimento informado.
Foram incluídas no estudo gestantes com idade entre 18 e 45 anos que não tinham filhos e que tivessem $\leq 24$ semanas de gestação, cujos fetos, depois de avaliação por meio de ultrassonografia, se mostraram vivos e morfologicamente normais. As gestantes foram separadas em dois grupos: Grupo AER (com história de dois ou mais abortos espontâneos de repetição) e o Grupo Controle (sem história de perdas gestacionais anteriores). Foram excluídas aquelas gestantes que já haviam sido submetidas a tratamento psicológico, psiquiátrico ou para transtornos sexuais antes da gravidez vigente ou durante esta, as que tinham sofrido um ou mais abortos não consecutivos e sucessivos, e as que não eram alfabetizadas ou que tinham dificuldade em compreender as questões dos instrumentos baseados em autorresposta.

No período de recrutamento, compareceram ao ambulatório 110 gestantes, e todas foram convidadas para participar do estudo. Quatro gestantes foram excluídas por terem abortos não consecutivos e uma por ter sido submetida a tratamento psiquiátrico previamente. Assim, das 105 gestantes, 55 compuseram o Grupo AER e 50 o Grupo Controle.

Foram aplicados os questionários sociodemográfico e de vivência da relação sexual durante a gravidez, autodesenvolvido, com questões autorresponsivas relacionadas a possíveis fantasias e sentimentos relacionados a perda do concepto, sangramento, dor e resposta sexual decorrente do ato sexual. Além destes, empregamos 3 testes Beck Depression Inventory (BDI) ${ }^{11}$, o qual se trata de uma escala de autorrelato que busca avaliar depressão e contém 21 itens, cada um deles com 4 alternativas com sintomas crescentes de depressão. Foi usado o Female Sexual Funtion Index (FSFI) ${ }^{12}$, questionário com 19 questões que abordam 6 domínios e que busca mensurar e avaliar a função sexual na pesquisa clínica que envolve gestantes. As gestantes responderam aos questionários em local reservado, tendo sua privacidade garantida.

As variáveis inicialmente foram descritas por cálculos de proporções para as variáveis categóricas e por média e desvio padrão para as variáveis contínuas. As variáveis categóricas foram comparadas entre os grupos por meio do teste do $\chi^{2}$ e as variáveis contínuas por meio do teste $t$ de Student (por apresentarem curvas de distribuição de normalidade). Foram considerados como nível de significância estatística valores de $\mathrm{p}<0,05$.

\section{Resultados}

A idade das entrevistadas variou entre 18 e 45 anos, com média e desvio padrão $(\sigma)$ entre $31,3( \pm 5,9)$ e 26,3( $\pm 5,4)$ anos nos Grupos AER e Controle, respectivamente. Houve diferença significante entre esses valores $(\mathrm{p}<0,05)$. Das pacientes do Grupo AER, $83 \%$ moravam 
com seus parceiros e 16,3\% eram solteiras. Entre as do Grupo Controle, esses percentuais eram, respectivamente, 48 e $52 \%(\mathrm{p}<0,05)$. A maioria das mulheres era branca (NS). O Grupo Controle apresentou maior nível educacional (NS). No entanto, a renda familiar mensal nos dois grupos foi de um salário mínimo $(\mathrm{p}=0,6)$. O tempo médio de gestação das mulheres do Grupo AER foi de 11,7 semanas e das do Grupo Controle, de 13,1, não havendo diferença significativa quanto à idade gestacional média dos grupos.

A Tabela 1, desenvolvida com base nos resultados obtidos mediante aplicação do Beck Depression Inventory $(\mathrm{BDI})^{11}$, mostra que a incidência de todos os níveis de depressão é aproximadamente duas vezes mais elevada entre as pacientes do Grupo AER, quando comparadas às do Grupo Controle (teste do $\chi^{2} ; \mathrm{p}<0,05$ )

A Tabela 2 apresenta os resultados referentes ao FSFI $^{12}$. O escore total foi respectivamente $16,4 \pm 9,1 \mathrm{e}$ $21,1 \pm 6,1$ para o Grupo AER e Controle, respectivamente. Ao analisar os diversos domínios, somente no do desejo não houve diferença significante entre os Grupos Estudo e Controle, respectivamente: 3, $4 \pm 1,3$ e 3,7 $\pm 1,1(p=0,1)$. Em todos os outros domínios, ou seja, na excitação,

Tabela 1. Beck Depression Inventory. depressão avaliada nas pacientes dos grupos com aborto espontâneo de repetição e controle (foi verificado, por meio do teste do $\chi^{2}$, que essa diferença é estatisticamente significante)

\begin{tabular}{lcccc}
\hline & \multicolumn{3}{c}{ AER } & \multicolumn{2}{c}{ Controle } \\
\cline { 2 - 5 } & $\mathrm{n}$ & $\%$ & $\mathrm{n}$ & $\%$ \\
\hline Mínimo & 22 & 40,0 & 37 & 74,0 \\
Leve & 22 & 40,0 & 8 & 16,0 \\
Moderado & 10 & 18,1 & 5 & 10,0 \\
Grave & 1 & 1,8 & 0 & - \\
Total & 55 & 100,0 & 50 & 100,0 \\
\hline
\end{tabular}

AER: aborto espontâneo de repetição.

Tabela 2. Escores total, médio e desvio padrão obtidos pelos Grupos com Aborto Espontâneo de Repetição e Controle no Female Sexual Function Index

\begin{tabular}{|c|c|c|c|}
\hline Domínio & $\begin{array}{l}\text { AER }(n=55) \\
\text { Média }(\sigma)\end{array}$ & $\begin{array}{c}\text { Controle }(\mathrm{n}=50) \\
\text { Média }(\sigma)\end{array}$ & Valor $p$ \\
\hline Desejo* & $3,4( \pm 1,3)$ & $3,7( \pm 1,1)$ & 0,1 \\
\hline Excitação\# & $3,1( \pm 1,9)$ & $3,9( \pm 1,4)$ & $<0,05$ \\
\hline Lubrificação" & $3,4( \pm 2,3)$ & $4,6( \pm 1,5)$ & $<0,05$ \\
\hline Orgasmo & $3,1( \pm 2,2)$ & $4,1( \pm 1,6)$ & $<0,05$ \\
\hline Sutisfação ${ }^{\dagger}$ & $3,3( \pm 2,3)$ & $4,5( \pm 1,8)$ & $<0,05$ \\
\hline Dor & $3,2( \pm 2,5)$ & $4,7( \pm 1,9)$ & $<0,05$ \\
\hline Total $^{\neq}$ & $16,4( \pm 9,1)$ & $21,1( \pm 6,1)$ & $<0,05$ \\
\hline
\end{tabular}

AER: aborto espontâneo de repetição; * Pontuação variando de 1.2 a b; \#Pontuação variando de 0 a 6 ; ${ }^{\dagger} A$ variação possível é de 0 até 6 ; : Pontuação variando de 1,2 a 36 com ponto de corte: escore total $\leq 26$ (risco para disfunção sexual). Nota: Valores p obtidos com o uso do teste $t$ de Student.

(Fonte: FSFI Questionnaire ${ }^{12}$.

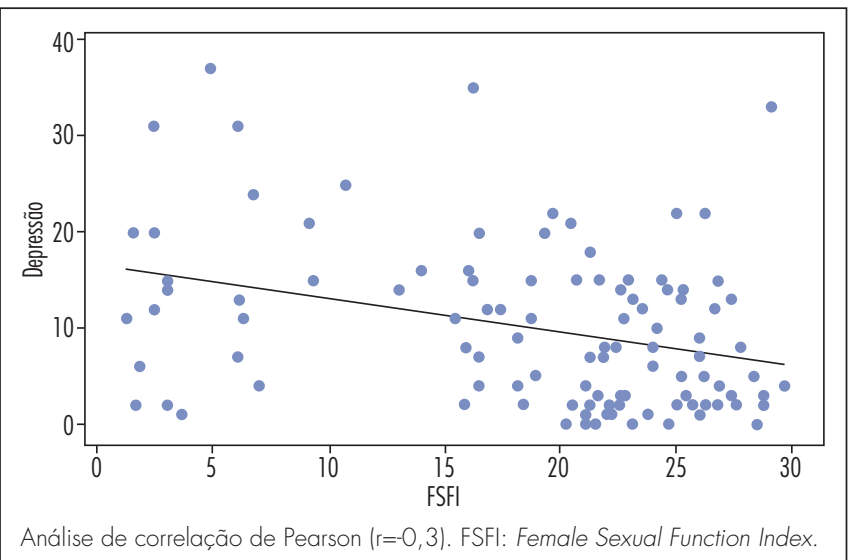

Figura 1. Correlação negativa entre depressão (Beck Depression Index) e sexualidade (Female Sexual Function Index) entre as 105 gestantes dos grupos com aborto espontâneo de repetição e controle.

lubrificação, orgasmo, satisfação e menor dor, os escores foram significativamente maiores no grupo sem AER.

Quando relacionamos os aspectos emocionais decorrentes da relação sexual na gravidez, em todas as questões encontramos diferenças significativas entre os grupos. As perguntas envolveram sentimentos relacionados a perda do feto, sangramento, dor e resposta sexual decorrente do ato sexual.

$\mathrm{Na}$ Figura 1, apresentamos a associação inversa entre depressão segundo BDI e função sexual segundo FSFI pela análise de correlação de Pearson $(r=-0,3)$. Ou seja, independentemente de a gestante ter ou não história de AER, quanto mais alto o escore de depressão, mais baixo o escore de sexualidade. Disso se conclui que todas as gestantes, quando estão deprimidas, apresenta maior risco de disfunção sexual.

\section{Discussão}

Mulheres que desenvolvem depressão durante a gravidez têm risco mais elevado de sofrerem complicações obstétricas e depressão pós-parto, o que é de grande relevância, pois esse quadro acarreta sequelas na relação materno-filial e nas relações socioculturais dessas mulheres ${ }^{13,14}$. Portanto, o ciclo gravídico-puerperal deve ser acompanhado a fim de se dar especial atenção à saúde mental das mulheres, identificando seus fatores de risco, principalmente a depressão, o transtorno mental de maior prevalência durante a gravidez e o puerpério.

Embora existam inúmeras ferramentas que possibilitem a quantificação da intensidade da depressão, o BDI ${ }^{11}$ foi eleito pela sua fácil aplicabilidade. Os resultados deste estudo mostraram que a presença de depressão moderada e grave é duas vezes mais frequente entre as mulheres com histórico de AER. Sua prevalência foi de $10,0 \%$ para as grávidas do Grupo Controle, que é próximo do que foi 
encontrado por Bennett et al. ${ }^{7}$ Já no Grupo AER, esse percentual foi de $19,9 \%$. Em outros estudos, são relatadas taxas de até $32 \%$ relacionadas a esse mesmo perfil de mulheres ${ }^{15,16}$. Quanto à depressão leve, sua prevalência foi de $40 \%$ entre as gestantes do Grupo AER e de $16 \%$ entre as do Grupo Controle. Isso nos leva a considerar a depressão como um aspecto relevante a se avaliar em estudos com gestantes com história de AER, e trabalhos recentes nos mostram a importância de se efetuar esse diagnóstico ainda durante a gestação, para a paciente ser tratada o quanto antes, reduzindo-se o risco de complicações no puerpério ${ }^{7}$.

Quanto à avaliação da função sexual, a ferramenta usada foi a FSFI ${ }^{12}$, validada na língua portuguesa, por ser este o questionário mais utilizado pela literatura internacional e que sumaria as várias fases da resposta sexual. Nossos dados mostraram que o comprometimento da sexualidade no Grupo AER é significativamente mais frequente do que no grupo sem AER. Somente no domínio do desejo os escores médios não apresentaram diferença significante, já que a redução desse escore foi observada nos dois grupos ${ }^{17-19}$.

Os estudos sobre a sexualidade têm mostrado que o transtorno do desejo, com a dor (disparenia) e o transtorno do orgasmo, são as queixas mais comuns no Brasil ${ }^{20,21} \mathrm{e}$ que aproximadamente um terço das mulheres em idade reprodutiva tem desejo hipoativo. Mimoun ${ }^{18}$ refere desejo hipoativo em uma em cada dez mulheres nos EUA e afirma que na Europa a prevalência é similar. Estudo feito no Brasil com grávidas com diabetes mellitus gestacional ${ }^{20}$ apresentou maior comprometimento do domínio do desejo nos dois grupos analisados. Outro estudo brasileiro feito com gestantes analisou que função sexual ruim gera piora relevante na qualidade de vida ${ }^{22}$.

Basson ${ }^{23}$, em seu novo modelo, propõe mover o foco do desejo sexual espontâneo (primeira fase do modelo trifásico de Kaplan), pois considera que a maioria das mulheres perde o desejo após algum tempo de relacionamento. De acordo com esse modelo, o desejo se inicia na neutralidade sexual; a satisfação sexual e a intimidade emocional é que serão valorizadas no encontro a dois, tirando-se o foco da genitalidade. A ausência de desejo não significa disfunção sexual ${ }^{24}$.

Enumeramos as principais dúvidas acerca desse tema. Entendemos que a sexualidade está envolta em tabus, mitos e preconceitos que nos acompanham de geração em geração. Entre as gestantes com histórico de aborto espontâneo de repetição, a sexualidade fica comprometida, e vários estudos já demonstraram que pode haver um distanciamento dos casais, tanto interpessoal como sexual, no primeiro ano após o aborto ${ }^{9,25,26}$. Para muitos casais, a sexualidade e a fertilidade estão associadas, sendo o AER um evento de impacto na vida sexual ${ }^{27}$. Perguntamos às componentes dos grupos seus medos quanto à relação sexual (dor, excitação, lubrificação e orgasmo). Como resultado, verificamos que as grávidas do Grupo AER demonstraram duas a cinco vezes mais medos relacionados à fantasia de que o sexo pode prejudicar os fetos no início da gestação como observado em outras investigações ${ }^{5,19}$.

Alguns casais evitam atividade sexual por medo de prejudicar o feto ou até mesmo a mãe. No entanto, é relatado por vários autores ${ }^{28,29}$ que a relação sexual não interfere no curso da gravidez. A associação inversa entre os resultados do $\mathrm{BDI}^{11}$ e do $\mathrm{FSFI}^{12}$ também já foi descrita anteriormente $e^{25,28,30}$. A depressão é um fator de risco para a disfunção sexual - causa desinteresse, apatia e sensação de fadiga, e potencializa outros sintomas que acabam por comprometer o desejo sexual - e, por outro lado, o desempenho sexual insatisfatório pode levar à depressão e causar conflitos relacionais, ou seja, desencadeia um círculo cumulativo entre disfunção sexual e depressão, no qual uma pode ser causa ou consequência da outra.

Entendemos que este estudo foi elaborado com participantes de um mesmo nível socioeconômico, o que impede que os resultados aqui apresentados sejam extrapolados para mulheres de outros contextos culturais ou níveis socioeconômicos, tendo em vista o impacto já conhecido das diferenças socioeconômico-culturais sobre a sexualidade. Entretanto, por não ter encontrado estudos analisando a associação entre depressão e comportamento sexual em gestantes com histórico de AER, consideramos como ponto positivo do nosso trabalho a originalidade do tema, utilizando dois questionários validados. O questionário $\mathrm{BDI}^{11}$, que é de fácil compreensão e mundialmente conhecido, assim como o FSFI ${ }^{12}$, instrumento que foi de grande valia, pois permite avaliar a função sexual das grávidas de forma específica.

Concluímos, portanto, que grávidas com história de AER apresentam frequência duas vezes mais elevada dos sintomas de depressão moderada e grave. Elas mostram cinco vezes mais preocupação com prejuízo ao feto com o coito. Disso decorre a função sexual mais comprometida quando comparada às mulheres sem AER em quase todos os ciclos de resposta sexual, exceto no domínio do desejo, já que esse domínio em ambos os grupos se encontra comprometido. Ainda pudemos avaliar que há uma associação inversa entre depressão e função sexual. 
1. Read J. $A B C$ of sexual health: sexual problems associated with infertility, pregnancy, and ageing. BMJ. 1999;318(7183):587-9.

2. Geller PA, Klier CM, Neugebauer R. Anxiety disorders following miscarriage. J Clin Psychiatry. 2001;62(6):432-8.

3. Tsartsara E, Johnson MP. The impact of miscarriage on women's pregnancy-specific anxiety and feelings of prenatal maternalfetal attachment during the course of a subsequent pregnancy: an exploratory follow-up study. J Psychosom Obstet Gynaecol. 2006;27(3): 173-82.

4. Bergner A, Beyer R, Klapp BF, Rauchfuss M. Pregnancy after early pregnancy loss: a prospective study of anxiety, depressive symptomatology and coping. J Psychosom Obstet Gynaecol. 2008;29(2):105-13.

5. Fertl K, Bergner A, Beyer R, Klapp BF, Rauchfuss M. Levels and effects of different forms of anxiety during pregnancy after a prior miscarriage. Eur J Obstet Gynecol Reprod Biol. 2009; 142(1):23-9.

6. Zukerman B, Amaro H, Bauchner H, Cabral H. Depressive symptoms during pregnancy: relationship to poor health behaviors. Am J Obstet Gynecol. 1989;160(5 Pt 1):1107-11.

7. Bennett HA, Einarson A, Taddio A, Koren G, Einarson TR. Prevalence of depression during pregnancy: systematic review. Obstet Gynecol. 2004;103(4):698-709.

8. Pereira PK, Lovisi GM, Pilowsky DL, Lima LA, Legay LF. Depression during pregnancy: prevalence and risk factors among women attending a public health clinic in Rio de Janeiro, Brazil. Cad Saude Publica. 2009;25(12):2725-36.

9. Serrano F, Lima ML. Recurrent miscarriage: psychological and relational consequences for couples. Psychol Psychother. 2006;79(Pt 4):585-94.

10. Laumann EO, Paik A, Rosen RC. Sexual dysfunction in the United States: prevalence and predictors. JAMA. 1999;281(6):537-44.

11. Cunha JA. Manual da versão em português das Escalas Beck. São Paulo: Casa do Psicólogo; 2001.

12. Bayer AG, Zonagen Inc., Target Health Inc. [Internet]. FSFI Scoring Appendix [cited 2012 Jan 31]. Available from: http://www.fsfiquestionnaire.com/FSFI\%20Scoring\%20Appendix.pdf

13. Miller L. Postpartum depression. JAMA. 2002;287(6):762-5.

14. Camacho RS, Cantinelli FS, Ribeiro CS, Cantilino A, Gonsales BK, Braguittoni $E$, et al. [Psychiatry disorders in pregnancy and puerperium: classification, diagnosis and treatment]. Rev Psiquiatr Clín. 2006;33(2):92-102. Portuguese.
15. Klock SC, Chang G, Hiley A, Hill J. Psychological distress among women with recurrent spontaneous abortion. Psychosomatics. 1997;38(5):503-7.

16. Couto ER, Couto E, Vian B, Gregório Z, Nomura ML, Zaccaria R, et al. Quality of life, depression and anxiety among pregnant women with previous adverse pregnancy outcomes. Sao Paulo Med J. 2009; 127(4): 185-9.

17. Nappi RE, Martini E, Terreno E, Albani F, Santamaria V, Tonani S, et al. Management of hypoactive sexual desire disorder in women: current and emerging therapies. Int J Womens Health. 2010;2:167-75.

18. Mimoun S. [Hypoactive sexual desire disorder, HSDD]. Gynecol Obstet Fertil. 2011;39(1):28-31. French.

19. Babazadeh R, Najmabadi KM, Masomi Z. Changes in sexual desire and activity during pregnancy among women in Shahroud, Iran. Int J Gynaecol Obstet. 2013;120(1):82-4.

20. Ribeiro MC, Nakamura MU, Abdo CH, Torloni MR, Scanavino MT, Mattar R. [Pregnancy and gestational diabetes: a prejudicial combination to female sexual function?] Rev Bras Ginecol Obstet. $2011 ; 33(5): 219-24$. Portuguese.

21. Abdo C. Estudo da vida sexual do brasileiro. São Paulo: Bregantini; 2004.

22. Ferreira DQ, Nakamura MU, Souza E, Mariani Neto C, Ribeiro MC, Santana TG, et al. [Sexual function and quality of life of low-risk pregnant women]. Rev Bras Ginecol Obstet. 2012;34(9):409-13. Portuguese.

23. Basson R. Human sex-response cycles. J Sex Marital Ther. $2001 ; 27(1) 33-43$.

24. Abdo $\mathrm{CH}$. Ciclo de resposta sexual: menos de meio século de evolução de um conceito. Diagn Tratamento. 2005; 10(4):220-2.

25. Bartellas E, Crane JM, Daley M, Bennett KA, Hutchens D. Sexuality and sexual activity in pregnancy. BJOG. 2000;107(8):964-8.

26. Hutti MH. Social and professional support needs of families after perinatal loss. J Obstet Gynecol Neonatal Nurs. 2005;34(5):630-8.

27. Nelson CJ, Shindel AW, Naughton CK, Ohebshalom M, Mulhall JP. Prevalence and predictors of sexual problems, relationship stress, and depression in female partners of infertile couples. J Sex Med. 2008;5(8):1907-14.

28. Soifer R. Psicologia da gravidez, parto e puerpério. 6a ed. Porto Alegre: Artes Médicas; 1980.

29. Suplicy M. Conversando sobre sexo. 3a ed. Rio de Janeiro: Vozes; 1983.

30. Fabre LF, Smith LC. The effect of major depression on sexual function in women. J Sex Med. 2012;9(1):231-9. 